\title{
Knowledge, behaviour and attitudes on induced abortion and family planning among Sri Lankan women seeking termination of pregnancy
}

\section{Jennifer Perera ${ }^{1}$, Tharangi de Silva ${ }^{2}$ and Harshana Gange ${ }^{2}$}

(Index words: Inadequate family planning services, legal status of pregnancy termination)

\begin{abstract}
Introduction In Sri Lanka over 500 induced abortions are done daily in spite of restrictive legislation. Experiences in other countries show that liberal laws alone have not solved the issues of induced abortions which may harm a woman's physical and mental health.
\end{abstract}

Objective To determine the socio-demographic features of women seeking termination of pregnancy, and their knowledge, attitude and behaviour with respect to induced abortion and family planning.

Methods A prospective study on a randomly selected group of 210 women attending a clinic in Colombo requesting termination of pregnancy. A pre-tested interviewer-administered questionnaire was used for data collection.

Results Over $80 \%$ of women seeking abortion were between 20 and 40 years of age. All religions were represented. $13 \%$ were single and $10 \%$ wanted to postpone a pregnancy. $38.6 \%$ had three or more children. In $90 \%$ the period of gestation was less than 10 weeks. Presence of a young child was the commonest reason for termination, followed by poverty. Only $0.9 \%$ were due to incest and foetal abnormality. $96 \%$ were not aware of adverse effects of abortion. $91 \%$ thought that induced abortion was immoral and $94 \%$ did not know that it was illegal. $29 \%$ had previous terminations and post-abortion contraception counselling was poor. Although $78 \%$ were knowledgeable on at least one method of contraception, only $16.3 \%$ were using it regularly.
Discussion A majority used induced abortion as a family planning method. Improving accessibility and the quality of family planning services is of paramount importance. Every encounter of a woman with a health care worker should be an opportunity for counselling.

\section{Introduction}

It is estimated that in Sri Lanka over 500 illegal induced abortions (IA) are done daily [1-3]. It is also estimated that 70 to 89 deaths per 100000 live births are due to unsafe abortions [4]. In Sri Lanka 5\% to $6 \%$ of all maternal deaths are due to abortion [5], and IA is illegal except when it is performed to save the mother's life. During the past few years many organisations have been lobbying for more liberal laws on abortion. However, an attempt to reform the law to permit termination for foetal malformation and pregnancy following rape or incest failed when the 1995 Penal Code Amendment was withdrawn. 'Right to life' arguments and other justifications based on tradition were articulated in the parliamentary debate [6].

Inadequate, inaccessible, and unaffordable family planning (FP) services and social barriers that prevent women and couples from using FP methods may be responsible for a substantial proportion of unwanted pregnancies. Even when FP services are available and accessible, a proportion of unwanted pregnancies arise

${ }^{1}$ Associate Professor, ${ }^{2}$ Temporary Lecturer, Department of Microbiology, Faculty of Medicine, University of Colombo, Sri Lanka. Correspondence: JP, Tel: +94 11 2697513, e-mail: microcmb@sltnet.lk (Competing interests: none declared). Received 5 May 2002 and revised version accepted 14 February 2004. 
following contraceptive failure due to incorrect use. Sri Lanka appears to have a well established FP program. The information collected by the Family Health Bureau (FHB) through the routine reporting system shows that in both state and non-state sector services the new acceptor rate of FP per 1000 married women, has increased from 57.6 in 1991 to 74.6 in 1996 [5]. But no data are available on the regularity of use of FP or dropout rates.

Data on IA are mostly extrapolated from health statistics and from hospitalised patients following sepsis; detailed information on IA is sparse. The information on the details of women seeking pregnancy termination will permit the design of effective policies, programs and strategies to address the issues of IA. In a recent study in Sri Lanka the socio-demographic data of IA seekers in 1997 was analysed [3]. Behaviour and attitude with regard to induced abortion, their knowledge of FP and factors responsible for poor use of family planning are additional issues that require attention.

\section{Methods}

A prospective study was done from August to October 1999 on a randomly selected group of 210 women attending a clinic in Colombo. Data were obtained using a pre-tested questionnaire administered by an interviewer after obtaining verbally expressed consent. The interview was conducted at the first visit for the present episode and before any counselling. Confidentiality was strictly maintained throughout the study. The data were analysed by EpiInfo version 6 statistical package. Approval for the study was obtained from the Faculty of Medicine, University of Colombo, Ethics Committee.

\section{Results}

The youngest subject was 14 years and the oldest was 47 . Over $80 \%$ were in the 20 to 40 year age group (Table 1). The level of education was higher than that of the general population [7] as shown in Table 2, and 95\% knew that sexual intercourse would result in pregnancy.

There were women of all religions, $77 \%$ were Buddhists, 9\% Roman Catholics and Christians, 4.3\% Hindus, 3.3\% Muslims, and the remaining 6.2\% were from non-traditional religious sectors. They were

Table 1. Age distribution of the study population $(n=210)$

\begin{tabular}{lc}
\hline Age (years) & Number of women (\%) \\
\hline $14-19$ & $18(8.6)$ \\
$20-29$ & $84(40.0)$ \\
$30-39$ & $88(41.9)$ \\
$\geq 40$ & $20(9.5)$ \\
\hline
\end{tabular}

Table 2. Level of education among abortion seekers $(n=210)$

\begin{tabular}{lcc}
\hline Level of education & Number $(\%)$ & $\begin{array}{c}\text { (\%) in the general } \\
\text { population [7] }\end{array}$ \\
\hline No schooling & $3(1.4)$ & 11.2 \\
Up to grade 8 & $74(35.2)$ & 69.8 \\
Up to O Level & $78(37.1)$ & 13.4 \\
Up to A Level & $54(25.7)$ & 14.8 \\
University & $1(0.5)$ & 0.7 \\
\hline
\end{tabular}

proportionate to that of the general population except for Hindus. The majority, 74.8\% (157/210), were not employed outside home. Factory workers accounted for $10 \%$ (21/ $210)$. There were four qualified nurses $(2.0 \%)$ and five schoolteachers $(2.4 \%)$.

The average income of the study group was less than the average for the general population [8]. The monthly income of the majority (63\%) was less than Rs. 5000 (approx. US \$50.00); 32\% were earning between Rs 5000 to 10000 monthly and only $3.6 \%$ were earning more than Rs 10 000. Most (86.6\%) were married, but $13 \%$ were single women. $23.3 \%$ did not have any children. Among the 49 who did not have children, 22 (44.9\%) were married and wanted to postpone having children. Over one-third of the women had three or more children. The data are summarised in Table 3. In the majority (90\%) the period of gestation (POG) was less than 10 weeks. However, $3.8 \%$ had a POG of $\geq 13$ weeks. $98.6 \%$ (207/ 210) of the partners knew about the pregnancy and $97.6 \%$ $(202 / 210)$ agreed to the termination.

Table 3. Number of children of termination seekers $(n=210)$

\begin{tabular}{lc}
\hline Number of children & Number of women (\%) \\
\hline None & $49(23.3)$ \\
1 & $34(16.2)$ \\
2 & $46(21.9)$ \\
$\geq 3$ & $81(38.6)$ \\
\hline
\end{tabular}

Reasons for the present termination are given in Table 4. Poverty, too soon after the last child, too old to have children, or being single were common reasons. In nearly one-third $(32 \%)$, the reason for termination was the presence of a young child. There were only $0.9 \%$ cases of induced abortion due to incest and foetal abnormality. There were no cases of rape.117 (56\%) were relieved that they would be able to terminate the pregnancy whereas $24(11.5 \%)$ were sad, and $68(32.5 \%)$ were indifferent to the termination at the time of interview. $96 \%$ of the women were not aware of the long term consequences of abortion such as pelvic inflammatory disease and subfertility. Among the initial 56 women 
interviewed $91 \%$ (51/56) thought that they were doing something immoral but resorted to abortion as there was no alternative, and 94\% (53/56) did not know that abortion was illegal. These two questions had to be removed from the questionnaire later at the request of the service provider. 88 women $(42.1 \%)$ were informed about the pregnancy termination clinic by their family doctor or another medical officer, 80 (38.3\%) were informed by persons who had attended the clinic previously, and $41(19.6 \%)$ by friends.

Table 4. Reasons for the present termination $(n=203)$

\begin{tabular}{lr}
\hline Reason & Number $(\%)$ \\
\hline Last child is too young & $66(32.5)$ \\
Poverty & $43(21.1)$ \\
Regulations for employment overseas & $26(12.8)$ \\
Large gap between this last child & $26(12.8)$ \\
Not physically or mentally fit for another child & $14(6.9)$ \\
Single & $14(6.9)$ \\
Career / studying & $5(2.5)$ \\
Spouse does not want another child & $4(2.0)$ \\
Schooling & $3(1.5)$ \\
History of foetal abnormality & $1(0.5)$ \\
Incest & $1(0.5)$ \\
\hline
\end{tabular}

\section{Characteristics of women who opted for repeat terminations}

Sixty $(28.5 \%)$ had previous terminations. In 42 this was the second termination, in 11 the third, and in 7 the fourth. The methods used for termination of the previous pregnancy were suction or menstrual regulation (MR) in 44, instrumentation in 13 and medication in $3.42(70 \%)$ had attended the same clinic at least once for previous terminations. Among the 60 who had at least one previous termination, only $20 \%$ (12/60) had been informed regarding prevention of future pregnancies by the use of FP methods. But none were using an FP method regularly, which indicates poor post-abortion counselling. $13.8 \%$ (8/60) suffered from abdominal pain and bleeding which sometimes continued for up to 2 months. None required hospitalisation.

\section{Knowledge and practice of FP}

Of the 210 women, $159(77.6 \%)$ knew at least one method of FP. Among the 159, 65.8\% knew about oral contraceptives, $53.1 \%$ of injectables (DMPA), $45.8 \%$ of condom, $28.6 \%$ of the intrauterine contraceptive device (IUCD), $18 \%$ of calendar method, $10.7 \%$ of tubal ligation and $3.9 \%$ of vasectomy. However, only two women (both nurses) were aware of post-coital contraception.

Of the 159 women, $45.3 \%$ women were introduced to FP by the public health midwife (PHM), $18.7 \%$ by media (television, newspaper) $15.4 \%$ by friends, $7.5 \%$ by the termination clinics, $11.7 \%$ by the family doctor and $1.2 \%$ by a hospital doctor. Among the 159, only $35.2 \%$ (56/159) had ever used an FP method, and among them only $16.3 \%(26 / 159)$ used a method regularly. Among the 30 who did not use a regular FP method, $80 \%$ did so due to sociocultural reasons (cost, privacy, accessibility were the main reasons), 16.7 due to adverse effects and $3.3 \%$ due to illness. The majority $42 \%$ obtained the FP devices from a pharmacy, $27.8 \%$ from a PHM, $16.7 \%$ (IUCD) from hospital, and $11.1 \%$ from a family practitioner. Among the 26 who claimed that they used a FP method regularly, $38.5 \%$ were using the calendar method, $30 \%$ the condom, $11.5 \%$ the contraceptive pill, 7.6\% DMPA injection and 3.8\% each the IUCD, Norplant and coitus interruptus.

\section{Discussion}

The present study confirms that the decision to terminate a pregnancy is largely independent of tradition, culture, religion and legal status of abortion [9]. In spite of IA being illegal, many women in Sri Lanka have access to termination through clinics in the private sector that are widespread in the island [3]. The commonest method used in these clinics for termination of early pregnancy is MR.

A majority of the women were not employed outside home. Among the employed groups, 10\% were factory workers. They were young and mostly single. They hailed from distant villages and lived in boarding houses away from their parents and guardians. These groups are at high risk and should be provided accurate information.

In Sri Lanka most pregnant women $(80 \%)$ register in antenatal clinics by fourth month of their pregnancy [5]. It would be appropriate to introduce FP information during the antenatal clinic visits. About $98 \%$ of the births in Sri lanka are hospital deliveries [10]. Women in the post-delivery period may be the most receptive group for advice on FP. A counselling service on FP should be made available at all maternity hospitals, so that the women have access to information before they leave the hospital or when they return to the well baby clinic at 6 weeks.

In the study group only $45 \%$ had been introduced to FP by the PHMW and among them a substantial number, $30 / 159$, had discontinued the FP method due to sociocultural reasons and adverse effects. There seems to be a failure in the follow up once FP is introduced in the primary care setting. The provision of FP advice and services by the PHM needs to be supplemented by other health care workers.

The majority (42\%) had to purchase FP devices from the pharmacy, and issues related to cost, privacy, accessibility, sociocultural acceptance contributed to noncompliance. The accessibility of FP devices should be improved. Among regular users of FP, a majority of the failures were in users of calendar method. Accurate information on correct use of the methods should be made available to prevent contraceptive failure. 
There is rekindled interest in lobbying for more liberal laws on abortion in Sri Lanka. Liberalising laws on abortion to include cases of rape, incest and foetal abnormality would not reduce the IA rate. This is because, the present study as well as the previous studies [3] have clearly shown, the number of IAs following rape, incest and foetal abnormality are small.

In India, abortion was made legal in 1972. Of the estimated 6.7 million abortions performed each year, 6.2 million abortions are done in non-governmental institutions, the majority under unsafe conditions. In 1994, $20 \%$ of maternal deaths in India were due to unsafe abortions [11].

In summary, prevention of unwanted pregnancies must be a high priority, and every attempt must be made to eliminate the need for IA by providing better FP services throughout the island. Family practitioners, nongovernmental organisations and private sector health care institutions should be recognised as important partners in the provision of FP services.

\section{References}

1. Wanigasundera M. Sri Lanka abortions cause concern. People 1984; 11: 37.

2. Abeywickrama D. KAP study on abortion in Sri Lanka. Family Planning Association of Sri Lanka. Colombo, Sri Lanka, 1988.
3. Ban DJ, Kim J, de Silva WI. Induced abortion in Sri Lanka: Who goes to providers for pregnancy termination? Journal of Biosocial Science 2002; 34: 303-15.

4. Unsafe abortions-global and regional estimates of incidence of and mortality due to unsafe abortions with a listing of available country data. 3rd edn. Division of Reproductive Health, Technical support WHO/RHT, WHO, Geneva, Switzerland, 1998.

5. Annual report on family health. Evaluation Unit, Family Health Bureau, Colombo, Sri Lanka, 1996: 3-6.

6. Gunasekera PC, Wijesinghe PS. Reducing abortions is a public health issue. Ceylon Medical Journal 2001; 46: 41-4.

7. Labour force and social economy survey. Census and Statistics Department 1992

8. Household income and expenditure survey. Census and Statistics Department 1995/1996. p 10-15.

9. Sandiuk A, Weingarten MA, Hart J. Contraception and religiousness in a general practice population in Israel. Family Practice 1984; 1: 37-41.

10. Annual report on family health. Evaluation Unit, Family Health Bureau, Colombo, Sri Lanka, 1999: pp. 4-6.

11. The safe motherhood action agenda: priorities for the next decade. Report on the safe motherhood technical consultation, 18-23 October 1997, Family Health Bureau, Colombo, Sri Lanka.

\section{Money, morals and the conquest of mortality (1)}

And it is money that is the problem. Money presumably began life as a convenience, as a portable means of trading that put an exchange medium in the place of barter. It began life as a symbol of value, but has become an abstraction against which value is measured. Money thus colonises our moral space.

It has also colonised political space almost completely. In Australia, for example, there's effectively only one viable political party. We might call it the Economic Realist Party. Like the recognised parties, it has its factions. Just to the left of centre is a faction which insists on emphasising a (heavily qualified) social awareness. To the right, a counterfaction espouses a (qualified) free-market philosophy. Both factions woo the corporate sector; both make gestures toward social welfare. Both temper their ideals with appeals to the central reality of economic restraints.

Miles Little. Keynote address at the Australian Health Care Summit 2003. Medical Journal of Australia 2003; 179: 432-3 